\title{
LOWER-BOUND LIMIT ANALYSIS OF MASONRY ARCHES WITH MULTIPLE FAILURE SECTIONS
}

\author{
NICOLA A. NODARGI ${ }^{*}$ AND PAOLO BISEGNA \\ Department of Civil Engineering and Computer Science \\ University of Rome Tor Vergata \\ via del Politecnico 1, 00133 Rome, Italy \\ e-mail: nodargi@ing.uniroma2.it (*corresponding author)
}

Keywords: Masonry Structures, Limit Analysis, Collapse mechanism, Arch, Linear Programming

\begin{abstract}
A computational method is proposed for the lower-bound limit analysis of masonry arches with multiple failure sections. Main motivation is the observation that, not only the position, but also the orientation of the failure sections in an arch might not be known in advance in practical applications. The lower-bound limit analysis problem is formulated as a straightforward linear programming problem. Numerical simulations highlight the predicting capabilities of the proposed approach, enabling an accurate and safe prediction of the loading capacity of masonry arches.
\end{abstract}

\section{INTRODUCTION}

The structural analysis of masonry structures plays a critical role in the effort to preserve and restore architectural heritage and historical buildings. Many computational approaches have been developed for addressing such a task, at different scales and levels of complexity, including micromechanical approaches (e.g., see [1,2]), multiscale/homogenization approaches (e.g., see [3-6]) and macromechanical/phenomenological approaches (e.g., see [711]), to be used in finite element formulations for inelastic structures (e.g., see [12-18]). As an alternative strategy, whose roots trace back to the first discovery by Robert Hook of the analogy between the structural behavior of a masonry arch and that of a catenary, the structural capacity can be computed by a limit analysis approach (e.g., see $[19,20])$.

In last decades, modern computers and advanced numerical methods have made possible to translate the limit analysis into a powerful tool for the analysis of masonry structures (e.g., see $[21,22]$. Restricting the attention to masonry arches, a successful approach is the rigid block computational method, originally conceived by Livesley [23], which regards the structure as a discrete system of rigid blocks exchanging unknown forces through their contact interfaces. The method has also been extended to three-dimensional structures (e.g, see [24,25]), nonassociative frictional behavior (e.g, see [26-29]) and large displacement regime (e.g, see [30,31]). In a different line of research, several computational approaches resort to the concept of thrust line for performing the lower-bound limit analysis of masonry arches (e.g., see [32-35]) Alternatively, in [36], it has been proposed to numerically integrate 

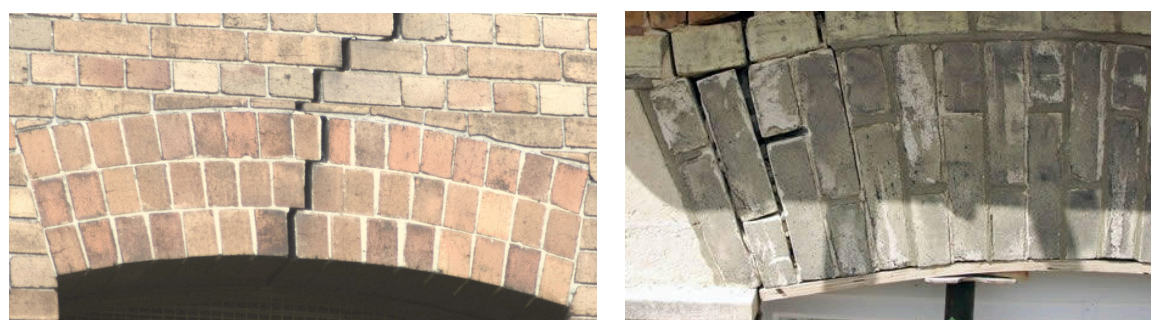

Figure 1: Collapse of masonry arches involving inclined failure sections with respect to the courses of the masonry units, hence calling for a generalization of the classical notion of arch with prescribed stereotomy.

Milankovitch's equilibrium equations [37,38], to characterize the equilibrated stress states of the arch, and then to proceed with the stress state optimization.

Interestingly, the methods mentioned above require, even if in disguise, that a stereotomy of the arch is clearly recognized for a spatial discretization of the problem. Indeed, as already pointed out by Milankovitch [37], the arch stereotomy does not simply allow for a reduction of the problem, but it rather affects its solution, because it implicitly defines the stereotomy sections as the potential failure sections, i.e. the sections where the opening of hinges can take place. Actually, no problem arises in the case of masonry arches constituted by individual voussoirs, as the stereotomy is naturally identified with the physical joints between them. Conversely, when the arch is monolithic or made by small units arranged in a regular or irregular texture, the selection of a stereotomy becomes contrived, and, for each possible

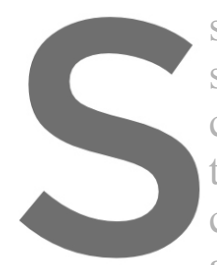
selection, a different so such observation, in concluded, and a variational the concept of the thrust line concept of stereotomy arbitrary position and orientation.
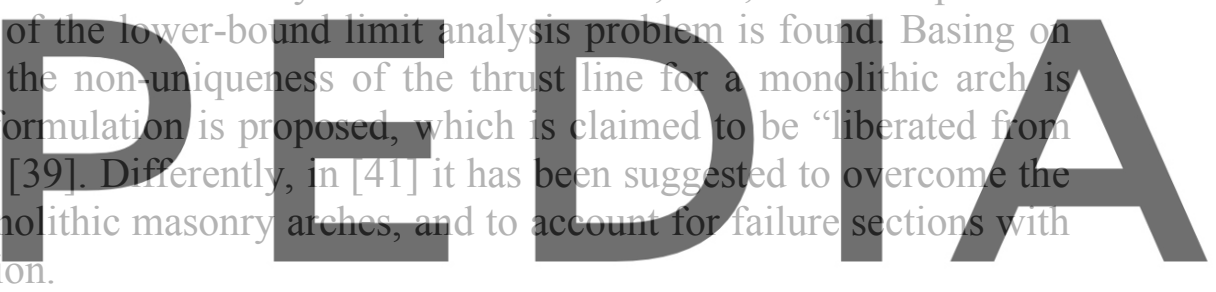

Aim of the present work is to propose a computational approach for the lower-bound limit

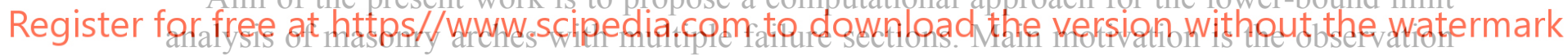

that not only the position, but also the orientation of the failure sections in an arch might not be known in advance in practical applications (Figure 1). Hence, provided a suitabie discrete number of potential failure sections is accounted for, the lower-bound limit analysis of a masonry arch is addressed by a straightforward LP problem. It is remarked that, considering multiple potential failure sections allows for an accurate description of the class of its equilibrated and statically admissible stress. Consequently, the resulting solution of the lowerbound limit analysis problem corresponds to the most dangerous stress state that might be induced by the external loads, or, from the dual kinematical standpoint, corresponds to the most dangerous collapse mechanism that might be induced in the structure by the opening of linear cracks. A safer prediction of the structural loading capacity is obtained compared to usual analysis, because taking into account a broader class of potential collapse states. Numerical simulations are presented for investigating the potentialities of the proposed computational approach.

The present paper is organized as follows. In Section 2 the lower-bound limit analysis of masonry arches with multiple failure sections is formulated. Numerical simulations are reported in Section 3 and conclusions are outlined in Section 4. 


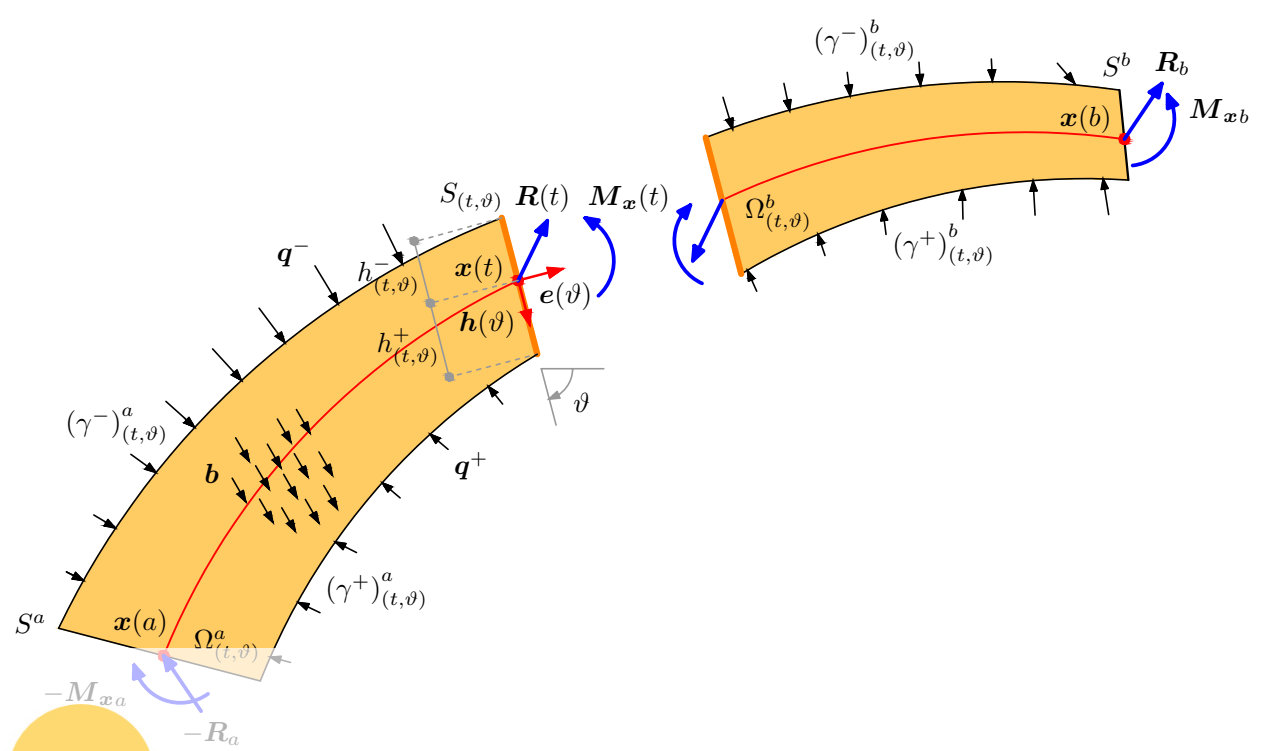

Figure 2: Masonry arch: geometric model, typical potential failure section and positive internal stress resultants are shown.

\section{FORMULATION}

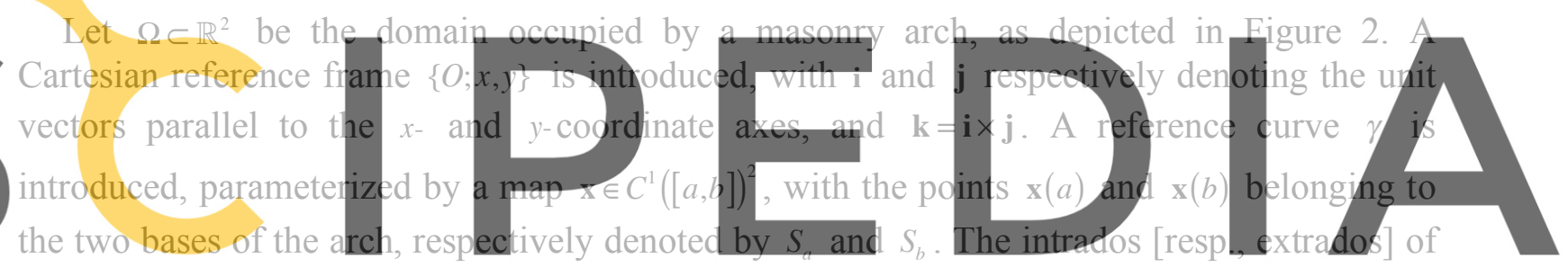

the arch is denoted by $\gamma^{+}\left[\right.$resp., $\left.\gamma^{-}\right]$. It is assumed that the arch is clamped at its bases $S_{a}$ and

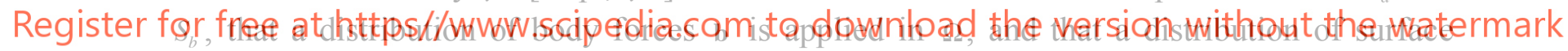

forces $\mathrm{q}^{+}\left[\right.$resp., $\left.\mathrm{q}^{-}\right]$acts over $\gamma^{+}\left[\mathrm{resp}, \gamma^{-}\right]$. In particular, those forces are expressed as $\mathbf{b}=\mathbf{b}^{\mathrm{d}}+\lambda \mathbf{b}^{1}$ and $\mathbf{q}^{ \pm}=\left(\mathbf{q}^{ \pm}\right)^{\mathrm{d}}+\lambda\left(\mathbf{q}^{ \pm}\right)^{1}$, i.e. split into the sum of dead and live contributions, the latter obtained by amplification of a basic distribution through the load multiplier $\lambda$. Furthermore, the arch is assumed to be comprised by a rigid no-tension material (e.g., see $[19,20])$.

A potential failure section of the arch $S_{(t, v)}$ is an oriented line segment, which contains the point $\mathbf{x}(t)$, has orientation defined by the polar angle $\vartheta$, and whose initial [resp., final] endpoint is located on the extrados [resp., intrados] curve. The unit vectors $\mathbf{h}(\vartheta)$ and $\mathbf{e}(\vartheta)$, respectively parallel and normal to $S_{(t, \vartheta)}$ are introduced. In addition, the signed distance of the endpoint of the section $S_{(t, \vartheta)}$ on $\gamma^{+}$[resp., $\gamma^{-}$], measured from $\mathbf{x}(t)$ along $\mathbf{h}(t)$, is denoted by $h_{(t, \vartheta)}^{+}\left[\right.$resp., $\left.h_{(t, \vartheta)}^{-}\right]$. It is noticed that the section $S_{(t, \vartheta)}$ cuts the arch into two portions, namely $\Omega_{(t, \vartheta)}^{a}$ and $\Omega_{(t, \vartheta)}^{b}$. Let $\left(\gamma^{ \pm}\right)_{(t, \vartheta)}^{a}$ and $\left(\gamma^{ \pm}\right)_{(t, \vartheta)}^{b}$ denote the portion of $\gamma^{ \pm}$bounding $\Omega_{(t, \vartheta)}^{a}$ and $\Omega_{(t, \vartheta)}^{b}$, respectively. 
The (internal) stress resultants $\mathbf{R}(t, \vartheta)$ and $\mathbf{M}_{\mathbf{x}}(t, \vartheta)$ at the potential failure section $S_{(t, \vartheta)}$ are defined by:

$$
\begin{aligned}
& \mathbf{R}(t, \vartheta)=\mathbf{R}_{a}-\int_{\Omega_{(t, \vartheta)}^{a}} \mathbf{b}(\mathbf{p}) \mathrm{d} a(\mathbf{p})-\int_{\left(\gamma^{ \pm}\right)_{(,, \vartheta)}^{a}} \mathbf{q}^{ \pm}(\mathbf{p}) \mathrm{d} \ell(\mathbf{p}), \\
& \mathbf{M}_{\mathbf{x}}(t, \vartheta)=\mathbf{M}_{\mathbf{x} a}+[\mathbf{x}(a)-\mathbf{x}(t)] \times \mathbf{R}_{a}-\int_{\Omega_{(t, \vartheta)}^{a}}[\mathbf{p}-\mathbf{x}(t)] \times \mathbf{b}(\mathbf{p}) \mathrm{d} a(\mathbf{p})-\int_{\left(\gamma^{ \pm}\right)_{(t, \vartheta)}^{a}}[\mathbf{p}-\mathbf{x}(t)] \times \mathbf{q}^{ \pm}(\mathbf{p}) \mathrm{d} \ell(\mathbf{p}),
\end{aligned}
$$

where $\mathbf{R}_{a}$ and $\mathbf{M}_{\mathrm{x} a}$ respectively denote the opposite of the clamp constraint force and moment reactions at the basis $S_{a}$, henceforth intended as the static indeterminate reactions of the problem.

The following component-wise representation of the stress resultants is introduced:

$$
N(t, \vartheta)=\mathbf{R}(t, \vartheta) \cdot \mathbf{e}(\vartheta), \quad T(t, \vartheta)=\mathbf{R}(t, \vartheta) \cdot \mathbf{h}(\vartheta), \quad M_{\mathbf{x}}(t, \vartheta)=\mathbf{M}_{\mathbf{x}}(t, \vartheta) \cdot \mathbf{k},
$$

where $N(t, \vartheta), T(t, \vartheta)$ and $M_{x}(t, \vartheta)$ can be respectively interpreted as normal force, shear force and bending moment at the potential failure section $S_{(t, \vartheta)}$

Following the model of no-tension material, the stress resultants $\mathbf{R}(t, \vartheta)$ and $\mathbf{M}_{\mathbf{x}}(t, \vartheta)$ acting over the potential failure section $S_{(t, 8)}$ have to obey suitable static admissibility conditions. Specifically, unilateral conditions are enforced:

$N(t, \vartheta) \leq 0, \quad-M_{\mathrm{x}}(t, \vartheta)+N(t, \vartheta) h_{(t, \vartheta)}^{+} \leq 0, \quad M_{\mathrm{x}}(t, \vartheta)-N(t, \vartheta) h_{(t, \vartheta)}^{-} \leq 0$,

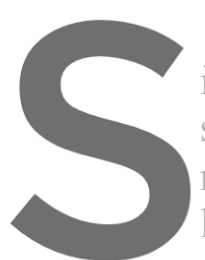

implying the normal force to be compressiv

material. As customary

large friction angle is

The static theorem of limit analysis require
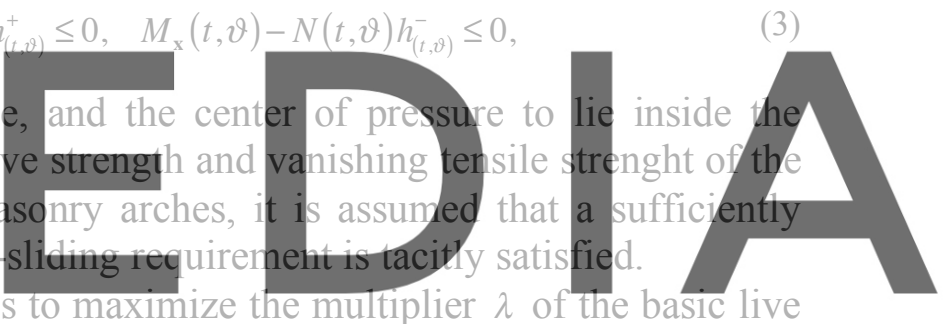

loads over the space of equilibrated and statically admissible stress resultants. Indeed,

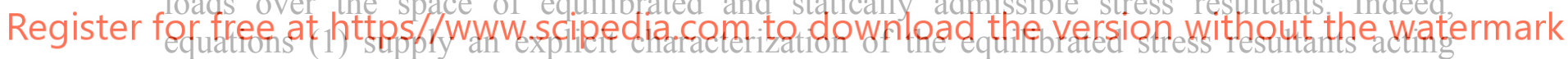

over the section $S_{(t, \vartheta)}$ in terms of the statically indeterminate reactions $\mathbb{R}_{a}$ and $\mathbf{M}_{\mathbf{x} a}$. The latter

are component-wise collected in the following vector:

$$
\mathbf{X}=\left\{H_{a}, V_{a}, M_{\mathbf{x} a}\right\},
$$

where $H_{a}=\mathbf{R}_{a} \cdot \mathbf{i}, V_{a}=\mathbf{R}_{a} \cdot \mathbf{j}, M_{\mathrm{x} a}=\mathbf{M}_{\mathrm{x} a} \cdot \mathbf{k}$, and the semicolon denotes column stacking. On the other hand, by exploiting positions (2), the equilibrated stress resultants (1) can be substituted in the static admissibility conditions (3), which boil down to linear inequalities in the unknowns $\mathbf{x}$ and $\lambda$. Hence, the lower-bound limit analysis problem for a masonry arch with multiple failure sections reduces to:

$$
\begin{aligned}
& \max _{\lambda, \mathbf{x}} \lambda, \\
& \text { s.t. } \quad \text { inequalities (3) hold for any } t, \vartheta .
\end{aligned}
$$

Indeed, for developing a computational solution strategy of problem (5), a suitable discretization is undertaken with respect to the parameters $t$ and $\vartheta$. That amounts at checking 
the static admissibility conditions only for a discrete number, say $C$, of potential failure sections, referred to as control sections. Hence, problem (5) can be finally written in the form:

$$
\begin{aligned}
& \max _{\lambda, \mathbf{X}} \lambda, \\
& \text { s.t. } \quad \mathbf{A X}-\mathbf{b}^{1} \lambda-\mathbf{b}^{\mathrm{d}} \leq 0,
\end{aligned}
$$

where $\mathbf{A}$ is a $3 C \times 3$ matrix, and $\mathbf{b}^{\mathrm{d}}$ and $\mathbf{b}^{1}$ are $3 C \times 1$ vectors (for details on the assembling procedure, see [41]). Problem (6) is a Linear Programming (LP) problem, whose solution can be addressed by standard optimization tools.

\section{NUMERICAL SIMULATIONS}

A pointed arch is considered, as depicted in Figure 3(a). Its geometry is characterized by the radius $R$, the eccentricity $e$, the embrace angle $\beta$ and the thickness $h$. The intrados and extrados curves of the arch are the union of two arcs of circle, having radius $R \pm h / 2$, center at point of coordinates $( \pm e,-R \cos (\beta / 2))$ and central angle $\beta / 2$, measured from the vertical direction. In order to mimic a pseudo-static earthquake loading, it is assumed that the arch is subjected to the self-weight (dead load) and to a distribution of horizontal forces proportional to the self-weight (live load). It is also assumed that the arch is clamped at its springing sections.

The reference curve of the arch is defined as its mid-curve. For investigating how the selection of potential failure sections affects the problem solution, the following choices are considered (Figure 3(b))
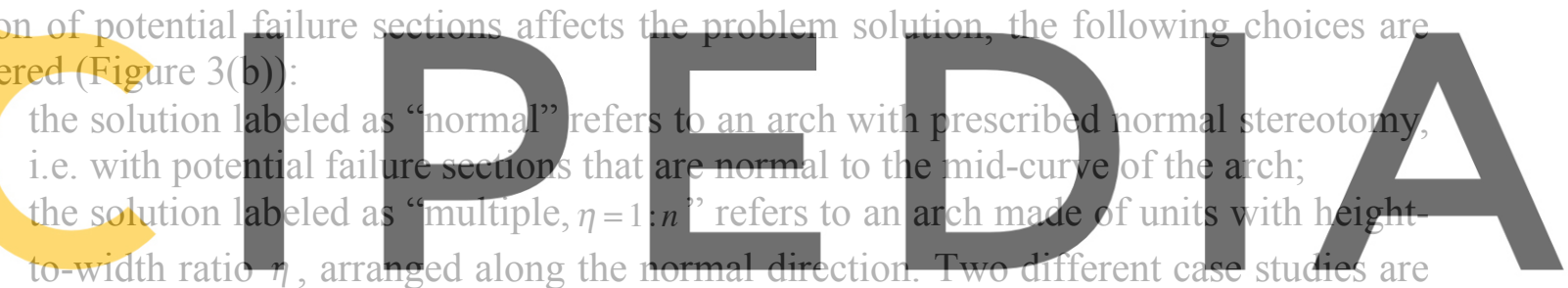

here considered, respectively with $n=2$ and $n=4$.

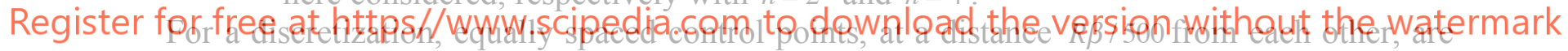

first considered along the reference curve of the arch. For the solution with normal stereotomy, normal control sections are selected passing through the control points. For the solution with multiple potential failure sections, a discrete set of orientation angles, geometrically compatible with the texture of the masonry, is preferred to generate potential failure sections. Accordingly, in addition to control sections parallel to the normal direction, also control sections with inclination angles $\vartheta_{k}= \pm \arctan (1 /(n / 2+k n)), k=\{0,1\}$, with respect to the normal direction, are considered [41] (Figure 3(b)).

As a preliminary step, a minimum thrust analysis has been conducted to compute the minimum thickness-to-radius ratio $h_{\min } / R$ of the pointed arch for the two values of the eccentricity-to-radius ratio $e / R=\{0.4,0.6\}$ at varying of the embrace angle $\beta$. Relevant results are respectively reported in Tables 1 and 2. Values corresponding to solutions with normal stereotomy are in agreement with the ones reported in [42]. It is noticed that slightly larger values of the minimum thickness-to-radius ratio $h_{\min } / R$ are required when multiple potential failure sections are taken into account. 
(a)

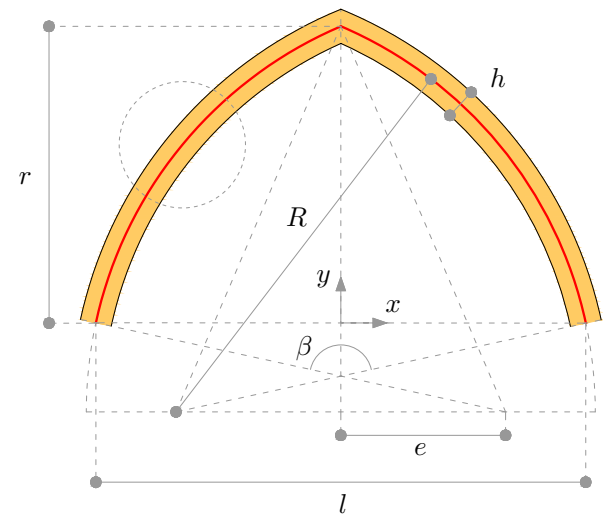

(b)

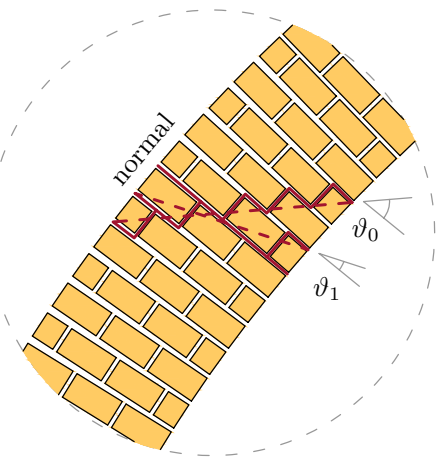

Figure 3: Pointed arch: (a) geometry, with highlighted reference curve, and (b) schematic illustration of the relationship between masonry texture and preferred (i.e. geometrically compatible) orientations of potential failure sections in the solution labeled as "multiple" (units with height-to-width ratio $\eta=1: 2$ are considered).

Table 1: Pointed arch: minimum thickness-to-radius ratio $h_{\min } / R$ for pointed arches with eccentricity-toradius radio $e / R=0.4$ and embrace angle $\beta$, assuming normal stereotomy or multiple potential failure sections.

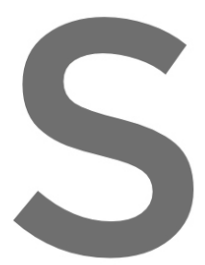

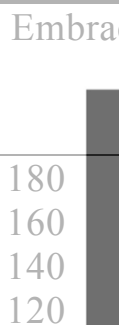

100

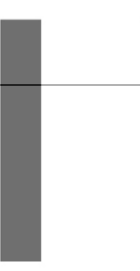

Register for free at http\$ $8 /$ www.scipedia.com

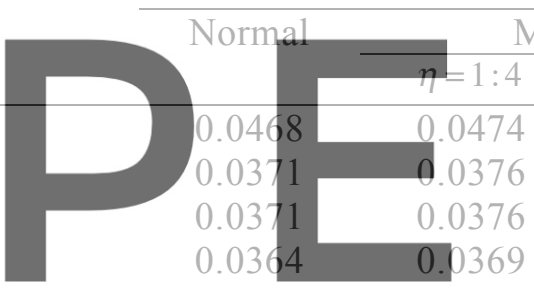

0.0316

0.0320
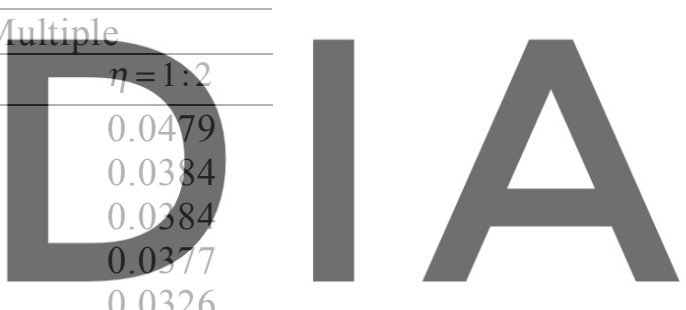

$0.0226 \quad 0.0228 \quad 0.0232$

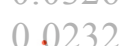

Table 2: Pointed arch: minimum thickness-to-radius ratio $h_{\min } / R$ for pointed arches with eccentricity-toradius radio $e / R=0.6$ and embrace angle $\beta$, assuming normal stereotomy or multiple potential failure sections.

\begin{tabular}{lccc}
\hline Embrace angle $\beta\left[^{\circ}\right]$ & \multicolumn{2}{c}{ Normalized minimum thickness $h_{\min } / R$} \\
\cline { 2 - 4 } & Normal & \multicolumn{2}{c}{ Multiple } \\
\cline { 2 - 4 } & & $\eta=1: 4$ & $\eta=1: 2$ \\
\hline 180 & 0.0391 & 0.0401 & 0.0417 \\
160 & 0.0390 & 0.0401 & 0.0417 \\
140 & 0.0388 & 0.0398 & 0.0414 \\
120 & 0.0337 & 0.0346 & 0.0360 \\
100 & 0.0226 & 0.0231 & 0.0240 \\
80 & 0.0054 & 0.0054 & 0.0051 \\
\hline
\end{tabular}




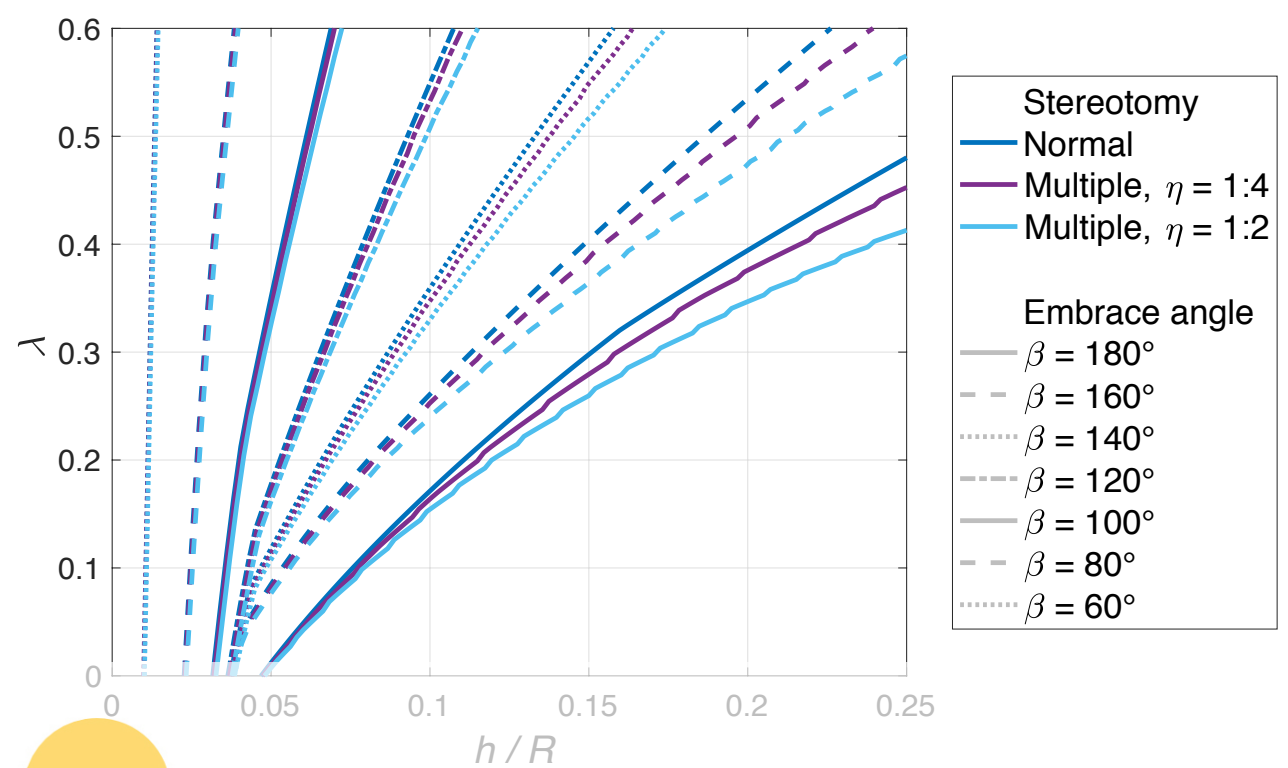

Figure 4: Pointed arch: horizontal forces collapse multiplier $\lambda$ versus thickness-to-radius radio $h / R$ for pointed arches with eccentricity-to-radius ratio $e / R=0.4$ and embrace angle $\beta$, assuming normal stereotomy or multiple potential failure sections.
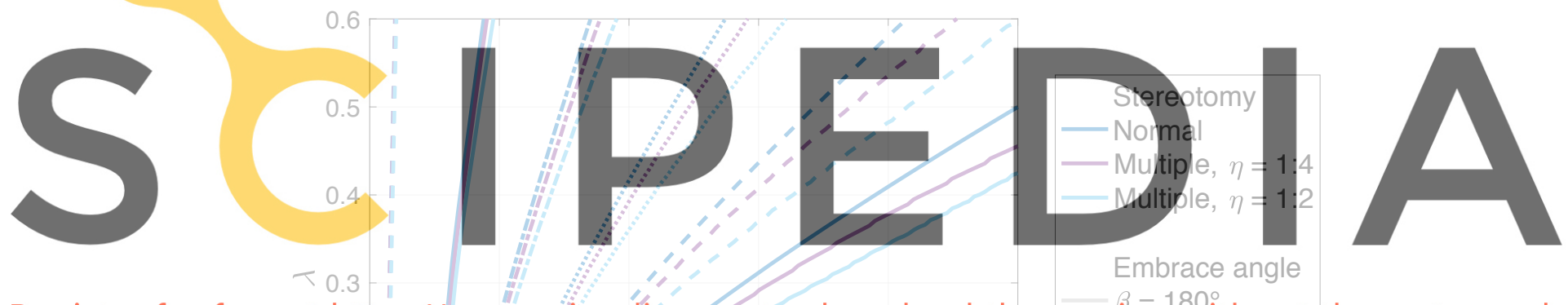

Register for free at https//www.secipedia.eorn to download the versibon without the watermark

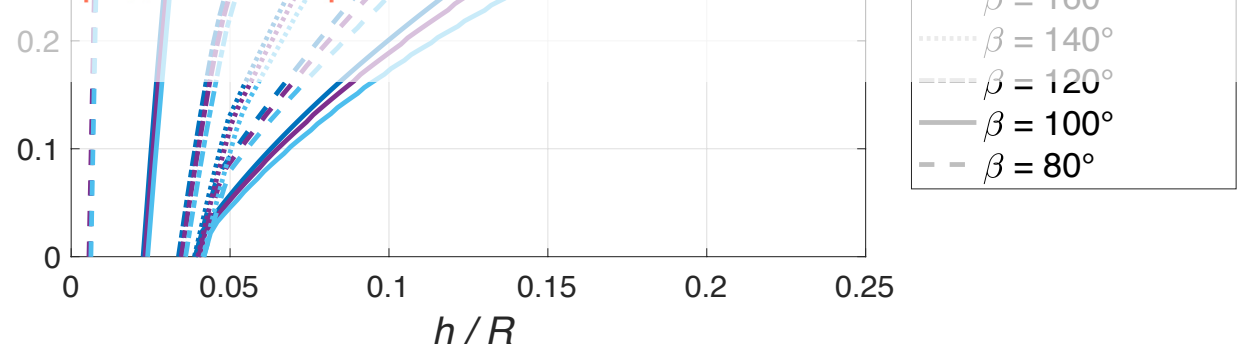

Figure 5: Pointed arch: horizontal forces collapse multiplier $\lambda$ versus thickness-to-radius radio $h / R$ for pointed arches with eccentricity-to-radius ratio $e / R=0.6$ and embrace angle $\beta$, assuming normal stereotomy or multiple potential failure sections.

A parametric analysis on the collapse multiplier $\lambda$ of the horizontal forces, as a function of the thickness-to-radius ratio $h / R$, has been carried out for the values of the eccentricity-toradius ratio $e / R=\{0.4,0.6\}$, at varying of the embrace angle $\beta$. The results are shown in Figures 4 and 5. 
(a1)

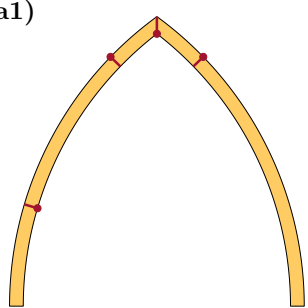

$(\mathrm{a} 2)$

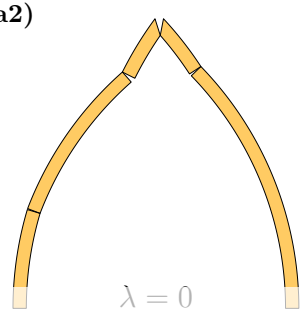

(b1)

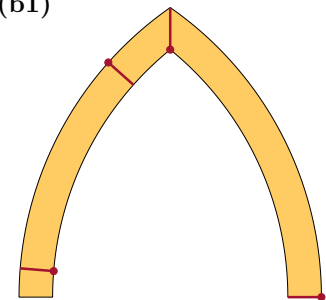

(b2)

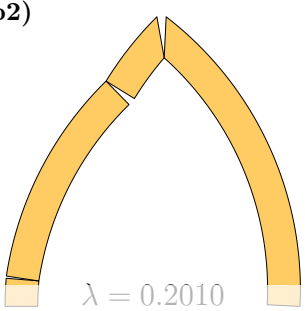

(c1)

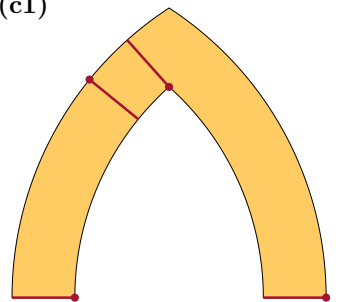

(c2)

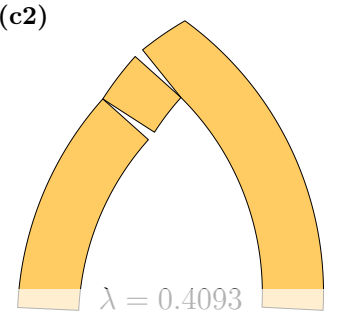

Figure 6: Pointed arch: (a) actual failure sections (first row) and collapse mechanism (second row) under

horizontal forces proportional to self-weight, assuming normal stereotomy. Eccentricity-to-radius ratio

e/ $R=0.6$, embrace angle $\beta=180^{\circ}$, and thickness-to-radius radio (a) $h / R=h_{\min } / R=0.0391$, (b) $h / R=0.10$ and (c) $h / R=0.20$ are considered.

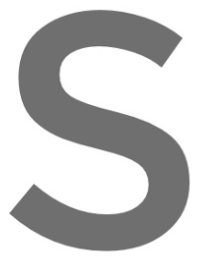

(a1)

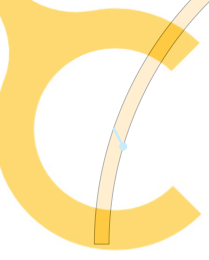

(a2) (b1)
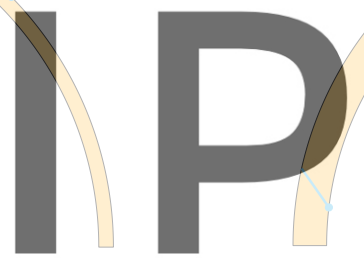

(b2)
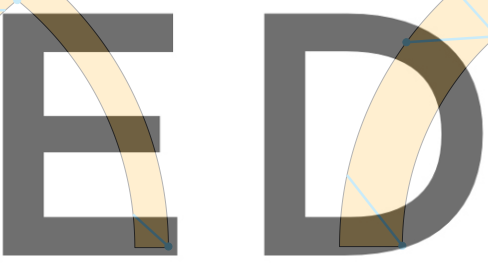

(c2)

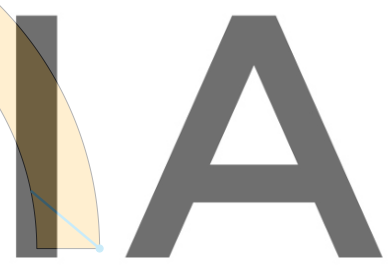

Register for free at https//www.scipedia.com to download the version without the watermark
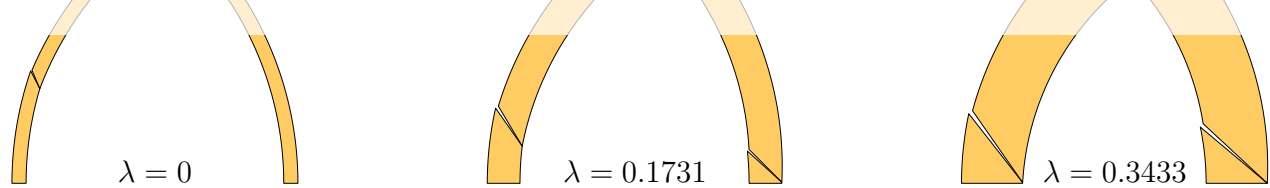

Figure 7: Pointed arch: (a) actual failure sections (first row) and collapse mechanism (second row) under horizontal forces proportional to self-weight, assuming multiple potential failure sections (units with height-towidth ratio $\eta=1: 2$ ). Eccentricity-to-radius ratio $e / R=0.6$, embrace angle $\beta=180^{\circ}$, and thickness-to-radius radio (a) $h / R=h_{\min } / R=0.0417$, (b) $h / R=0.10$ and (c) $h / R=0.20$ are considered.

As already noted in [42] in regards to the solution with normal stereotomy, for given values of $e / R$ and $\beta$, a two-branches curve describes the collapse multiplier $\lambda$ as a function of the thickness-to-radius ratio $h / R$. In particular, the first branch, corresponding to small thickness-to-radius ratios $h / R$, is associated to a four-hinge collapse mechanism in which no right-springing hinge is involved, Figure 6(a). Conversely, a right-springing hinge characterizes the second branch of the curve, corresponding to moderately large or large 
thickness-to-radius ratios $h / R$. In that case, for increasing thickness-to-radius ratios $h / R$, the most-left hinge smoothly migrates towards the left springing of the arch, with the collapse mechanism shifting from one as in Figure 6(b) to one as in Figure 6(c). Indeed, for small embrace angles $\beta$, the second branch of the curve is not developed at all.

Concerning the solution with multiple failure sections, the curves of $\lambda$ versus $h / R$ have the same general trend as the ones pertaining to the solution labeled as "normal". In fact, collapse mechanisms that are qualitatively similar take place, i.e. four-hinge collapse mechanisms with right-hinge springing or not depending on $h / R$, as depicted in Figure 7. However, the fact that the actual failure sections may have different orientations with respect to the normal direction implies that the solution with multiple potential failure sections is associated to a reduced loading capacity of the arch with respect to the solution with normal stereotomy. The reduction of the loading capacity of the arch predicted in the case of multiple potential failure sections results to be more pronounced for larger values of the height-towidth ratio $\eta$ of the units (i.e., for less elongated units), and may be in the order of $15 \%$. As an increase in $\eta$ is related to an increase in the maximum inclination angle of the potential failure sections with respect to the normal direction, that suggests the maximum inclination angle of the potential failure sections to be a decisive parameter in the determination of the loading capacity of the arch.

In closing, it is remarked that the selection of potential failure sections in the arch has a significant influence in the prediction of its collapse states. Except for arches that are made of

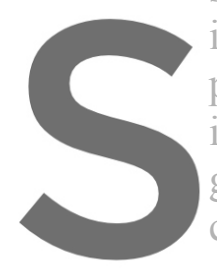
individual voussoirs, prescribed stereotomy is to take into considera geometrically compati collapse states that requirements need to be fulfilled by the arch.
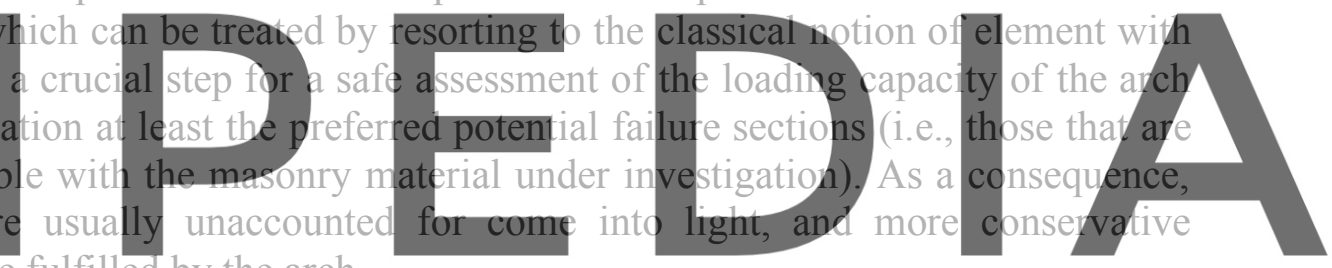

Register for freatultsps/ywww.scipedia.com to download the version without the watermark

A computational method has been proposed for addressing the lower-bound limit analysis of masonry arches with multiple potential failure sections. That model is conceived to generalize the classical notion of masonry arch with prescribed stereotomy, which results insufficient in applications where the orientation of the failure sections, in addition to their position, is not a priori known. In fact considering multiple potential failure sections amounts at taking into account a broader class of potential collapse mechanisms induced by the opening of linear cracks with multiple potential positions and orientations. Provided a finite number of control sections is considered in the arch, the lower-bound limit analysis problem has been formulated as a straightforward linear programming problem. Numerical simulations, also dealing with benchmark problems, have been presented for highlighting the predicting capabilities of the proposed methodology.

\section{REFERENCES}

[1] Gambarotta, L. and Lagomarsino, S. Damage models for the seismic response of brick masonry shear walls. Part I: the mortar joint model and its applications. Earthq. Eng. 
Struct. Dyn. (1997) 26(4):423-439.

[2] Oliveira, D.V. and Lourenço, P.B. Implementation and validation of a constitutive model for the cyclic behaviour of interface elements. Comput. Struct. (2004) 82(17-19):14511461 .

[3] Milani, G. Simple homogenization model for the non-linear analysis of in-plane loaded masonry walls. Comput. Struct. (2011) 89(17-18):1586-1601.

[4] Addessi, D. and Sacco, E. A multi-scale enriched model for the analysis of masonry panels. Int. J. Solids Struct. (2012) 49(6):865-880.

[5] Petracca, M., Pelà, L., Rossi, R., Oller, S., Camata, G. and Spacone, E. Regularization of first order computational homogenization for multiscale analysis of masonry structures. Comput. Mech. (2016) 57(2):257-276.

[6] Braides, A. and Nodargi, N.A. Homogenization of cohesive fracture in masonry structures. Math. Mech. Solids (2020) 25(2):181-200.

[7] Pelà, L., Cervera, M. and Roca, P. Continum damage model for orthotropic materials: Application to masonry. Comput. Meth. Appl. Mech. Eng. (2011) 200(912):917-930.

[8] Pelà, L., Cervera, M. and Roca, P. An orthotropic damage model for the analysis of masonry structures. Constr. Build. Mater. (2013) 41:957-967.

[9] Nodargi, N.A., Artioli, E., Caselli, F. and Bisegna, P. State update algorithm for associative elastic-plastic pressure-insensitive materials by incremental energy minimization. Fracture and Structural Integrity (2014) 29:111-127. [10] Nodargi, N.A. and. Bisegna, P. State update algorithm for isotropic elastoplasticity by
incremental energy minimization. In . J. Numer. Methods Erg. (2015) 105(3): 63-196.
[11] Gatta, C., Addessi, D. and Vestroni, F. Static and dynamic nonlinear response of
masonry walls. Int. J. Solids Struct. (2018) 155:291-303.
[12] Brasile, S., Casciaro, R. and Formica, G. Finite elennent fornulation for nonfinear
analysis of masonry walls.Comput. Struct (2010) 88(3-4):135-143.

[13] Cervera, M., Chiumenti, M. and Codina, R. Mixed stabilized finite element methods in

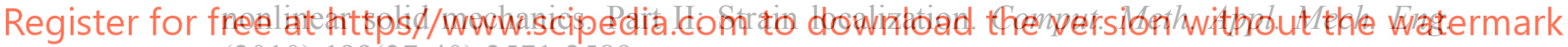
(2010) 199(37-40):2571-2589.

[14] Nodargi, N.A., Caselli, F., Artioli, E. and Bisegna, P. A mixed tetrahedral element with nodal rotations for large-displacement analysis of inelastic structures. Int. J. Numer. Methods Eng. (2016) 108(7):722-749.

[15] Nodargi, N.A. and Bisegna, P. A novel high-performance mixed membrane finite element for the analysis of inelastic structures. Comput. Struct. (2017) 182:337-353.

[16] Nodargi, N.A. An overview of mixed finite elements for the analysis of inelastic bidimensional structures. Arch. Comput. Method Eng. (2019) 26(4):1117-1151.

[17] Nodargi, N.A. and Bisegna, P. A mixed finite element for the nonlinear analysis of inplane loaded masonry walls. Int. J. Numer. Methods Eng. (2019) 120(11):1227-1248.

[18] Nodargi, N.A. and Bisegna P. A mixed membrane finite element for masonry structures. In: Carcaterra A. et al. (Eds.): Proceedings of XXIV AIMETA Conference 2019. AIMETA 2019, Lecture Notes in Mechanical Engineering. Springer (2020), pp. 1167-1178.

[19] Heyman, J. The stone skeleton. Int. J. Solids Struct. (1966) 2(2):249-279.

[20] Como, M. Statics of historic masonry constructions. Springer-Verlag (2016).

[21] Roca, P., Cervera, M., Gariup, G. and Pelà, L. Structural analysis of masonry historical constructions. Classical and advanced approaches, Arch. Comput. Method Eng. (2010) 
17(3):299-325.

[22] Tralli, A., Alessandri, C. and Milani, G. Computational methods for masonry vaults: a review of recent results, Open Civ. Eng. J. (2014) 8:272-282.

[23] Livesley, R.K. Limit analysis of structures formed from rigid blocks. Int. J. Numer. Methods Eng. (1978) 12(12):1853-1871.

[24] Portioli, F., Casapulla, C. and Cascini, L. An efficient solution procedure for crushing failure in 3D limit analysis of masonry block structures with non-associative frictional joints. Int. J. Solids Struct. (2015) 69-70: 252-266.

[25] Intrigila, C., Nodargi, N.A. and Bisegna P. Frictional behaviour of masonry interfaces: experimental investigation on two dry-jointed tuff blocks. In: Carcaterra A. et al. (Eds.): Proceedings of XXIV AIMETA Conference 2019. AIMETA 2019, Lecture Notes in Mechanical Engineering. Springer (2020), pp. 2032-2047.

[26] Baggio, C. and Trovalusci, P. Limit analysis for no-tension and frictional threedimensional discrete systems. Mech. Based Des. Struct. Mach. (1998) 26(3):287-304.

[27] Ferris, M.C. and Tin-Loi, F. Limit analysis of frictional block assemblies as a mathematical program with complementarity constraints. Int. J. Mech. Sci. (2001) 43(1):209-224.

[28] Gilbert, M., Casapulla, C. and Ahmed, H.M. Limit analysis of masonry block structures with non-associative frictional joints using linear programming. Comput. Struct. (2006) 84(13-14):873-887.

[29] Nodargi, N.A., Intrigila, C. and Bisegna, P. A variational-based fixed-point algorithm for the limit analysis of dry-masonry block structures with non-associative Coulomb friction. Int. J. Mech. Sci. (2019) 161-162:105078.

[30] Portioli, F. and Cascini, L. Large displacement analysis of dry-jointed masonry structures subjected to settlements using rigid block modelling. Eng. Struct. (2017) 148:485-496.

[31] Intrigila, C., Nodargi, N.A. and Bisegna P. Square cross vaults on spreading supports. In: R. Aguilar et al. (Eds.): Structural Analysis of Historical Constructions, RILEM Bookseries 18 (2019), pp. 1045-1053.

[32] Block, P., Ciblac, T. and Ochsendorf, J. Real-time limit analysis of vaulted masonry buildings. Comput. Struct. (2006) 84(29-30):1841-1852.

[33] O'Dwyer, D.W. Funicular analysis of masonry vaults. Comput. Struct. (1999) 73(15):187-197.

[34] Marmo, F. and Rosati, L. Reformulation and extension of the thrust network analysis. Comput. Struct. (2017) 182:104-118.

[35] Nodargi, N.A. and Bisegna, P. Thrust line analysis revisited and applied to optimization of masonry arches. Int. J. Mech. Sci. (2020) 179:105690.

[36] Ricci, E., Fraddosio, A., Piccioni, M.D. and Sacco, E. A new numerical approach for determining optimal thrust curves of masonry arches. Eur. J. Mech. A-Solids (2019) 75: 426-442.

[37] Milankovitch, M. Theorie der druckkurven. Zeitschrift für Mathematik und Physik (1907) 55:1-27.

[38] Foce, F. Milankovitch's theorie der Druckkurven: Good mechanics for masonry architecture. Nexus Netw. J. (2007) 9(2):185-210.

[39] Alexakis, H. and Makris, N. Minimum thickness of elliptical masonry arches. Acta Mech. (2013) 224(12): 2977-2991. 
[40] Alexakis, H. and Makris, N. Limit equilibrium analysis of masonry arches. Arch. Appl. Mech. 85(9-10): 1363-1381.

[41] Nodargi, N.A. and Bisegna, P. A unifying computational approach for the lower-bound analysis of systems of masonry arches and buttresses. Submitted (2020).

[42] Cavalagli, N., Gusella, V. and Severini, L. Lateral loads carrying capacity and minimum thickness of circular and pointed masonry arches. Int. J. Mech. Sci. (2016) 115-116:645656. 